Astropart. Phys., submitted

\title{
Radiation fields of disk, BLR and torus in quasars and blazars: implications for gamma-ray absorption
}

\author{
Alina-C. Donea円 and R.J. Protheroe \\ Department of Physics and Mathematical Physics \\ The University of Adelaide, Adelaide, SA 5005, Australia
}

\begin{abstract}
The radiation fields external to the jets and originating from within a few parsecs from the black hole, are discussed in this paper. They are the direct radiation from an accretion disk in symbiosis with jets, the radiation field from the broad line region (BLR) surrounding the accretion disk, and the infrared radiation from a dusty torus. The jet/disk symbiosis modifies the energetics in the central parsec of AGN such that for a given accretion rate, a powerful jet would occur at the expense of the disk luminosity, and consequently the disk would less efficiently ionize the BRL clouds or heat the dust in the torus, thereby affecting potentially important target photon fields for interactions of gamma-rays, accelerated electrons and protons along the jet.

Motivated by unification schemes of active galactic nuclei, we briefly review the evidence for the existence of broad line regions and small-scale dust tori in BL Lacs and Fanaroff-Riley Class I (FR-I) radio galaxies. We propose that an existing jet-accretion disk symbiosis can be extrapolated to provide a large scalesymbiosis between other important dusty constituents of the blazar/FR-I family. In the present paper, we discuss in the context of this symbiosis interactions of $\mathrm{GeV}$ and $\mathrm{TeV}$ gamma-rays produced in the jet with the various radiation fields external to the jet in quasars and blazars, taking account the anisotropy of the radiation.
\end{abstract}

\section{PACS Codes:}

98.54.Aj Quasars

98.54.Cm Active and peculiar galaxies

98.70.Rz gamma-ray sources

\section{Keywords:}

accretion disk, broad line region, torus, gamma-ray, AGN, photon-photon pair production

\footnotetext{
${ }^{1}$ Corresponding Author: adonea@physics.adelaide.edu.au, fax: +61 883034380
} 


\section{Introduction}

Following the introduction of unification schemes for active galactic nuclei (AGN), it is now widely accepted that the central nucleus of an active galaxy has a Kerr black hole, a relativistic accretion disk, jets, a dusty torus and clouds emitting broad emission lines. Jets and accretion disks are strongly related through a symbiosis, and we postulate that there may also be a large scale symbiosis involving dust components. Since $\gamma$-rays are attenuated by photon-photon pair production collisions with lower energy photons from the disk, BLR, or torus, such a symbiosis could have an effect on the production and absorption of $\gamma$-rays in AGN as the BLR and torus are powered by the disk, and so may depend on the way in which the jets are fed by the accretion disk.

A $\gamma$-ray flare could occur, for example, when a significant amount of energy has been accumulated in the inner part of the accretion disk and is subsequently expelled into the jets. As a consequence, relativistic shocks may form in the jets and could account for the observed rapid and high amplitude $\gamma$-ray variability. The intense emission observed during a flare could be also due to an increase in the accretion rate, in the bulk Lorentz factor of the emitting material in the jet, or in the efficiency of particle acceleration. The production of $\gamma$-rays from relativistic jets depends also on the density of radiation fields since these photons can be scattered by relativistic electrons to $\mathrm{GeV}$ and $\mathrm{TeV}$ energies, the external radiation field giving additional target photons to the internal synchrotron radiation assumed in the synchrotron self-Compton (SSC) process [1, 2]. Mannheim [3] and Protheroe [4] have shown that the disk radiation can be relevant for $\gamma$-ray production if the main constituents of the jet are relativistic protons.

In this paper we shall concentrate on the role played by external radiation fields in the absorption of $\gamma$-rays by photon-photon pair production, and we shall calculate the pair production opacity for $\mathrm{GeV}-\mathrm{TeV} \gamma$-rays emitted in the jets of quasars and blazars. We will only consider external radiation fields existing in AGN, and will not discuss pair production by $\gamma$-rays interacting with internal radiation fields in the emission region as this has been discussed elsewhere [5, 6]. We shall also not address the problem of absorption by the infrared background radiation during propagation to Earth (e.g. refs. [7, 8]).

We shall assume that the disk radiation, the BLR radiation, and the torus radiation is anisotropic, but is symmetric about the jet axis. In such a radiation field the reciprocal of the mean interaction length for photon-photon pair production by $\gamma$-rays traveling along the jet axis is given by

$$
x_{\gamma \gamma}^{-1}(E, z)=\int_{\varepsilon_{\min }}^{\infty} d \varepsilon \int_{\cos \left(\theta_{\max }\right)}^{\cos \left(\theta_{\min }\right)} \frac{d n}{d \Omega}(\varepsilon, \theta, z)(1-\cos \theta) \sigma_{\gamma \gamma}(s) 2 \pi d \cos \theta
$$

where $\theta$ is the angle between the direction of the soft photons and the jet axis, $\sigma_{\gamma \gamma}(s)$ is the photon-photon cross section for pair production at center of momentum frame energy squared $s=2 E \epsilon(1-\cos \theta), d n / d \Omega$ is the differential photon number density, and $\cos \left(\theta_{\max }\right)$ and $\cos \left(\theta_{\min }\right)$ are defined by the disk, BLR, or torus geometry involved or, in the case of

$\cos \left(\theta_{\min }\right)$, by the pair production threshold condition. We calculate the optical depth for 
absorption of $\mathrm{GeV}$ and $\mathrm{TeV}$ photons from the point of $\gamma$-ray emission to infinity, in the relevant external radiation fields.

In quasars the torus and the BLR are active components, as can be inferred from the large forest of emission lines observed, and from the thermal infrared spectrum, allowing good estimation of the diffuse radiation within the central parsec. In contrast, blazars, which comprise BL Lacs and Optically Violently Variable (OVV) quasars, are a special category of AGN having the jet aligned closely to the line of sight. The lack of emission lines and UV bump in blazars is usually assumed to be due to a low thermal activity at the centre, implying that the energy density of the external radiation is small in blazars compared to that in quasars. We shall investigate whether or not this is the case, and discuss how the $\gamma$-ray output could be different in quasars and blazars depending on the dominant radiation field.

In Section 2 we shall discuss the optical/UV emission from an accretion disk in symbiosis with the jet, and its effect on the BLR emission clouds (ionized by disk photons) and the torus (heating of dust). It is difficult to estimate the disk luminosity in blazars as has been done for some quasars with visible UV bumps [9, 10]. Blazars do not show thermal disk emission, and their spectra from radio to UV frequencies, sometimes to Xray frequencies, are universally attributed to synchrotron emission from the relativistic jet. The nonthermal boosted radiation from the jet dwarfs any thermal emission, making the direct detection of BLR or torus activity, and hence direct measurements of external photon fields present within the parsec scale of blazars, impossible.

We assume that the accretion disk surrounding the black hole and the relativistic jets are related by a small-scale symbiosis [9, 11], and that the energy available for the jet is the energy which would be dissipated between the last marginally stable circular orbit (in Kerr geometry), $R_{\mathrm{ms}} \approx 1.23 R_{g}$ where $R_{g}=G M / c^{2}$ is the gravitational radius, and the radius of the base of the jet $R_{\text {jet }}>R_{\mathrm{ms}}$. The total power of the jet depends on $R_{\text {jet }}$. A thick jet base means that more energy is expelled into the jet, and therefore, less energy is dissipated in the disk. A direct consequence of this assumption is that an accretion disk in symbiosis with jets (ADJ) produces a disk luminosity smaller than that of a "standard" [12 relativistic accretion disk. We suggest that the ADJ model could describe low-luminosity accretion disks in some blazars, and may provide a bridge between the standard accretion disk assumed to exist in quasars and Fanaroff-Riley Class II (FR-II) sources [13 and the low-power advection dominated accretion disk which may be found in some FR-I sources.

In Section 3, assuming reasonable models for the size and distribution of BLR clouds, we calculate the energy density of the radiation field of the BLR, and the optical depth for absorption of $\mathrm{GeV}$ and $\mathrm{TeV}$ photons. Broad Line Regions surround accretion disks and may also be important sources of soft photons which could be inverse-Compton scattered by relativistic electrons in the jet to produce $\gamma$-rays in "external Compton models" [2].

In section 4, we discuss the infrared radiation from a dusty torus. Based on the assertion that FR-I and BL Lacs are related [14], and that there is strong evidence that some FR-I have dusty tori [15], we assume that blazars must also have dusty tori [16] and 
discuss what evidence there is to support this. Since the infrared emission produced by tori could inhibit the escape of $\mathrm{TeV} \gamma$-rays due to photon-photon pair-production [17] we study how changes in disk activity can modify the geometry and the heating mechanism of the torus, and how this affects the optical depth for $\gamma-\gamma$ absorption in the torus infrared radiation.

\section{Radiation from an accretion disk with jets}

Relativistic outflows are very nicely described by a model of the small-scale symbiosis between the accretion disk and jets [11]. Donea and Biermann [9] have used such a model for an accretion disk with jets starting at the inner region of a disk, and were able to reproduce the UV bump in quasars. By fitting observed spectra they derived upper limits for the radius of the base of the jet, $R_{\text {jet }}$, and they found that $R_{\text {jet }}$ cannot be too far from $R_{\mathrm{ms}}$. The jet base, or "footring", is actually the thin layer between $R_{\mathrm{ms}}$ and radius $R_{\text {jet }}$, the jet being approximated by a hollow cylinder of inner radius $R_{\mathrm{ms}}$ and outer radius $R_{\text {jet }}$, such that $R_{\mathrm{ms}} \leq R_{\text {jet }} \ll R_{\text {out }}$, where $R_{\text {out }}$ is the outer radius of the disk.

The ADJ model assumes that the gravitational potential energy available between $R_{\mathrm{ms}}$ and $R_{\text {jet }}$ is the energy reservoir of the jets. The total power of the jets is strongly dependent on the accretion mass rate in the disk and on the size of its footring (we not include here the interaction between the black hole and jets which could also result in it putting a non-negligible fraction of its energy into the jets [18]). The total power of the jets is

$$
Q_{\text {jet }}=L_{\text {disk }}-L_{\text {disk }}^{\text {jet }}
$$

where $L_{\text {disk }}^{\text {jet }}$ is the luminosity of the disk with jets and $L_{\text {disk }}$ would be the luminosity of the disk if the conditions necessary to drive outflows were not met, i.e., the case of a standard relativistic disk.

A coupled jet-disk system must obey the laws of conservation of mass and angular momentum. We assume that the jet is fed with mass by the accretion disk, and that the flow of mass into the jet per unit of time $\dot{M}_{\text {jet }}=q_{m} \dot{M}$ is a fraction $q_{m} \leq 1$ of the accretion mass rate into the disk $\dot{M}=-2 \pi R \Sigma u^{R}$, where $\Sigma$ is the surface density of mass in the disk and $u^{R}$ is the radial velocity of gas at given radius $R$. The equation for conservation of mass requires $\dot{M}=\dot{M}_{\text {disk }}+\dot{M}_{\text {jet}}$. Derivation of the relevant equations can be found in Donea and Biermann [9], who followed the standard method of calculating the emission spectrum from an accretion disk 12. If there is no angular momentum and mass loss into the jet the equations used for mass and angular momentum transport in the disk become the standard equations [12], with $R_{\text {jet }} \rightarrow R_{\mathrm{ms}}, Q_{\text {jet }} \rightarrow 0$ and $L_{\text {disk }}^{\text {jet }} \rightarrow L_{\text {disk. }}$. In this paper, we shall adopt $q_{m}=0.1$ as used by Falcke et al. [19] in interpreting the radio-UV correlation in AGN.

The local physics at the inner radius of the disk (radius $R_{\text {jet }}$ ) is directly related to the extraction of angular momentum from the in-falling gas, and so modifies the structure of the relativistic disk [20]. From this one calculates the dissipation energy at radius 
$R, D^{*}(R)$, which must be done numerically in the case of a Kerr black hole, and a detailed discussion of this would serve no useful purpose here. Instead, for the purpose of illustration, and for the sake of simplicity we give here only the relation for the simpler case of a Schwarzschild black hole:

$$
D^{*}(R)=\frac{3 G M \dot{M}}{8 \pi R^{3}}\left[\left(1-q_{m}\right)-\left(1-q_{m}\right)\left(\frac{R_{\text {jet }}}{R}\right)^{1 / 2}\right]
$$

where $q_{m}=\dot{M}_{\text {jet }} / \dot{M}$ and $M$ is the black hole mass; nevertheless, in the present paper we use $D^{*}(R)$ for the disk of a Kerr black hole. A reasonable outer radius of the footring of the jet would be $R_{\text {jet }} \leq 10 R_{\mathrm{g}} \approx 10^{-4} M_{8}$ pc where $M_{8}=M / 10^{8} M_{\odot}$.

The disk/jet symbiosis is reflected mainly in a modified photon spectrum from the inner region of the accretion disk where the jet is anchored. The important result is that the spectrum from a Kerr accretion disk is cut off at high frequencies, from extreme UV to soft X-rays. In Fig. [ 1 we plot disk luminosities for the ADJ model, versus the thickness of the footring of the jet for Kerr black holes with masses $M=10^{8} M_{\odot}$ and $M=10^{9} M_{\odot}$, and for different mass accretion rates given by $\dot{m}=\dot{M} / \dot{M}_{\text {edd }}$, where $M_{\text {edd }}$ is the Eddington accretion rate. The case $R_{\text {jet }}=R_{\mathrm{ms}}$ corresponds to an accretion disk without jets ("standard" accretion disk) and $Q_{\text {jet }}=0$. As can be seen, for thicker jet bases more energy is available to power jets (dotted curves show $Q_{\text {jet }}$ increasing with $R_{\text {jet }}-R_{\mathrm{ms}}$ ) and less energy is radiated by the disk (solid curves show $L_{\text {disk }}$ versus thickness). We note that a small variation of the geometry at the coupling between jet and disk at radii $R_{\text {jet }}$ less than $\sim 3 R_{g}$ would induce large variations in the power of the jet, possibly causing flare activity in blazars. This is because, in a Kerr metric, at small radii close to the black hole there is a large amount of gravitational potential energy available for dissipation into the jet.

Because of the jet/disk coupling, the ADJ model predicts a lower disk luminosity and a softer photon spectrum, and some of the implications of this for interactions of accelerated electrons or protons are discussed in a separate paper [21]. As mentioned earlier, a flaring state could arise from enhanced AGN central activity whereby the jets get more energy from the disk. In this case, the disk changes from an ADJ with weak jets into an ADJ with a spectrum cut off at high frequencies but with correspondingly stronger jets. Since, in this case, the average energy of photons from an ADJ with strong jets (flaring) becomes lower than the average energy of photons from an ADJ with weak jets this will change the shape of the resulting $\gamma$-ray spectrum in models [2, 22] where disk photons are inverse-Compton scattered by relativistic electrons in the jet.

Luminosities of disks in blazars are probably $L_{\text {disk }}<10^{46} \mathrm{erg} / \mathrm{s}$, while quasars typically have $L_{\text {disk }}=10^{46}-10^{48} \mathrm{erg} / \mathrm{s}$. The ADJ model gives a simplified approach to the symbiosis between the disk and jets, and could describe low-luminosity accretion disks for quasars and some blazars. However, for some blazars the ADJ model could still give UV fluxes much higher than those observed, and in this case an ejection dominated accretion flow (EDAF) model, which is an ADJ model with a wind [23], may be better. An EDAF model has been applied to the central activity of Sgr $\mathrm{A}^{*}$ where it was shown that a wind 


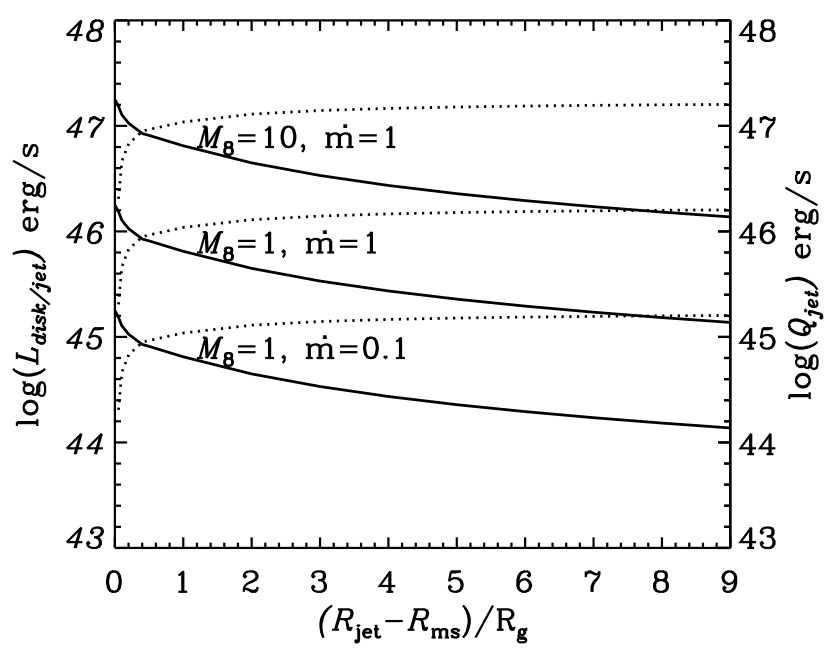

Figure 1: Luminosities of disks with jets vs. the thickness of the jet's footring $\left(R_{\text {jet }}-R_{\mathrm{ms}}\right)$ (solid curves). The dotted lines show $Q_{\text {jet }}$ vs. the thickness of the footring. $\left(M_{8} \equiv\right.$ $\left.M / 10^{8} M_{\odot}, q_{m}=0.1\right)$.

plus jets extracts energy from the disk more efficiency. Since the EDAF model does not leave much energy to be dissipated in the accretion disk, the radiation field from the disk would become even less important for $\mathrm{GeV}$ and $\mathrm{TeV} \gamma$-ray absorption. Alternatively, the jets could extract more energy from the accreting gas if the disk with a jets turns into an advection dominated accretion flow (ADAF) [24].

Fig. 2(a) shows the optical depths from $z=0.01$ pc to infinity for absorption of $\mathrm{GeV}-\mathrm{TeV}$ photons produced in the jet and interacting with photons from an ADJ with $q_{m}=0.1$. We see that at $1 \mathrm{TeV}$ photons are only absorbed for high black hole masses and mass accretion rates close to the Eddington limit. This could apply to quasars where the disk radiation field has a high density around the base of the jet. For low accretion mass rates with $\dot{M} \leq 0.1 M_{\text {edd }}$, and lower black hole masses, $M \leq 10^{8} M_{\odot}$, the photon-photon optical depth is $\tau_{\gamma \gamma}<1$ at $1 \mathrm{TeV}$. We note that the disk luminosity corresponding to the case $\dot{m}=1$ for $M_{8}=1$ lies roughly at the boundary between blazars and quasars. In Fig. 2(b) we plot the optical depth divided by $L_{46} \equiv L_{\mathrm{UV}} / 10^{46} \mathrm{erg} \mathrm{s}^{-1}$, and give the likely range for blazars and quasars using $\dot{m}=1$ for $M_{8}=1$ as the boundary. In blazars we may have ADJ models with accretion rates as low as $\dot{m}=0.01-0.001-$ at lower accretion rates an ADAF or EDAF model including jets may be more realistic. We use $M_{8}=1$ and $\dot{m}=0.01$ as the low luminosity boundary of blazars with an ADJ in Fig. 2(b). Similarly, we use a supermassive black hole $\left(M_{8}=10\right)$ and accretion close to the Eddington limit $(\dot{m}=1)$ for the upper luminosity boundary of quasars in Fig. 2(b). Of course, these boundaries are by no means rigid and there will be some overlap between the two populations, particularly when we plot $\tau_{\gamma \gamma} / L_{46}$. 

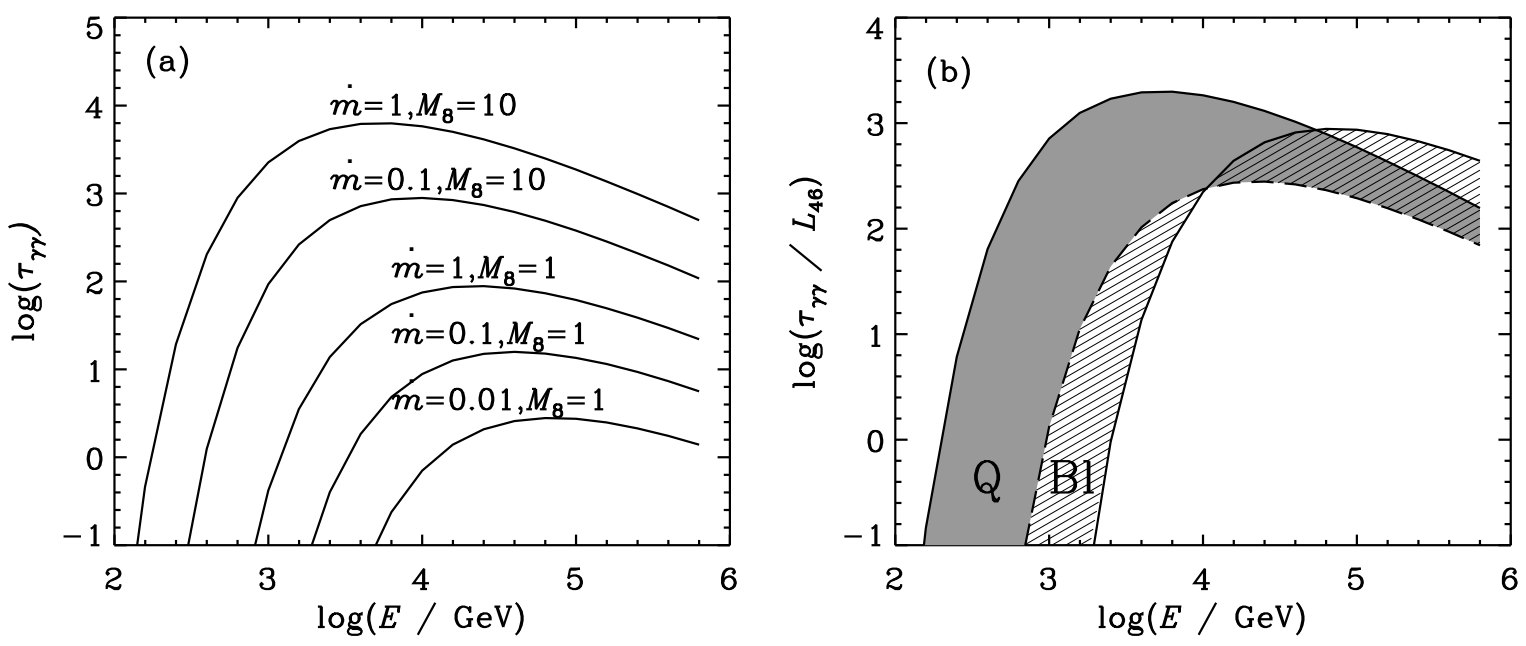

Figure 2: Optical depths from $z=0.01 \mathrm{pc}$ to infinity for $\gamma$-rays produced in the jet interacting with UV disk photons for the case of an ADJ model with $q_{m}=0.1$ and $R_{\text {jet }}=10 R_{g}$ : (a) for various black hole masses and accretion rates indicated by the labels attached to the curves; (b) optical depth normalized to disk luminosity in units of $10^{46}$ erg $\mathrm{s}^{-1}$ showing the probable range of $\tau_{\gamma \gamma}$ for blazars "Bl" and quasars "Q". 
The ADAF model seems to explain better very low luminosity blazars, but it cannot energize the BLR or heat efficiently dust in the torus; the lack of observed BLR and torus activity for some blazars has been sometimes interpreted as suggesting that blazars do not have tori or BLR. We consider that if blazars are to be included in the unification schemes they should also have BLR and dusty tori, but possibly with lower emission than in quasars, and we shall discuss this possibility later.

\section{Photons from broad line region clouds}

We are interested in obtaining the optical depth for absorption of $\mathrm{GeV}$ and $\mathrm{TeV}$ photons in the anisotropic radiation field of the BLR. For this we need to know the distribution of the BLR clouds around the jet axis. Broad line regions in AGN are not spatially resolved, but information about their size and the gas distribution can be obtained by reverberation mapping, from which it appears that BLR are stratified, and have typical sizes of the order of light-weeks in Seyfert galaxies and light-months in quasars [25].

Despite the fact that the majority of BL Lacs do not show any emission lines, there are observations suggesting that there is a weak broad $H_{\alpha}$ emission line in BL Lacertae itself [26], and even Mrk 421 seems to have a very low BLR luminosity, $L_{\mathrm{BLR}}=1.5 \times 10^{40} \mathrm{erg} / \mathrm{s}$ [27]. Interestingly, Celotti et al. [28] have suggested, based on the rapid TeV variability of Mrk 421, that its accretion rate is lower than $10^{-2}-10^{-3}$ of the Eddington rate. Hence, a less efficient ADJ or an ADAF may then power the BLR for this source. For Mrk 501, the BLR luminosity has been found to be $L_{\mathrm{BLR}}=4 \times 10^{41} \mathrm{erg} / \mathrm{s}$ [29]. In addition, Chiaberge et al. [30] have concluded that BLR and obscuring tori are closely linked in quasars, and both are present only in association with accretion.

In order to model the BLR, and hence its radiation field, we need to know its size and the emission line emissivity as a function of radius. The two-phase (hot/cold) model of the BLR predicts a relatively thin shell-like BLR [31]. Higher ionization lines are found at smaller radii (e.g. for NGC 5548 [32]), and this may favour a thin shell model for the BLR. This provides a very simple model of the BLR in which the total BLR region luminosity $L_{\mathrm{BLR}}$ is emitted from the surface of a sphere of radius $r_{\mathrm{BLR}}$, i.e. $L_{\mathrm{BLR}} / 4 \pi r_{\mathrm{BLR}}^{2}$ per unit area.

Although it is not known if strong winds can exist in blazars, there are models which explain the formation of clouds from bloated stars exposed to winds from accretion disks [33, 34], and shocks from jets could also contribute to cloud formation [5]. On the other hand, Frometh and Melia [35] suggest that the unsteady, turbulent accretion flow onto the black hole is subjected to several disturbances capable of producing shocks, which would heat the gas and allow for formation of BLR clouds. They also suggest that circular clouds originate at smaller radii. Results from reverberation mapping 25 suggest that the BLR in Seyferts is extended with an outer radius at least 10 times larger than the inner radius, and Kaspi and Netzer [36] use models with a radial dependence of BLR cloud number density and radius to fit their data.

Clearly, there are several possible mechanisms for formation of BLR clouds and it is 
not known which one operates in blazars. It could be that all these mechanisms play a role in BLR cloud formation. We shall adopt two extreme cases for the distribution of BLR cloud emissivity: (i) a geometrically thin shell at radius $r_{\mathrm{BLR}}$, (ii) a geometrically thick shell extending from radius $r_{\text {in,BLR }}$ to $r_{\text {out,BLR }}$ with the radial dependence of line emissivity based on the best fit parameters of Kaspi and Netzer [36] used in their modelling of the BLR in NGC 5548 (number density of clouds $n_{\mathrm{cl}} \propto r^{-1.5}$, cloud radius $R_{\mathrm{cl}} \propto r^{0.6}$ ). In both cases we shall assume that the line emission is isotropic, and that the ionization source (the accretion disk) is located at $r=0$ and has luminosity $L_{\mathrm{UV}}$. Accretion disk photons excite the BLR material, and emission lines such as $H_{\alpha}, H_{\beta}$, OIII, NII, etc., are produced. We simplify our problem by making the approximation that the entire BLR luminosity is emitted in the $H_{\alpha}(6563 \AA)$ line.

The BLR is assumed to be optically thin with optical depth $\tau_{\text {BLR }}$ such that the total BLR luminosity is $L_{\mathrm{BLR}} \approx \tau_{\mathrm{BLR}} L_{\mathrm{UV}}$. Celotti et al. [37] analyzed a sample of quasars and found an average value of $L_{\mathrm{BLR}} \approx 4 \times 10^{45} \mathrm{erg} / \mathrm{s}$, and for a sample of 12 blazars (including Mrk 421 and Mkr 501) found an average value of $L_{\mathrm{BLR}} \approx 2 \times 10^{43} \mathrm{erg} / \mathrm{s}$. However, flat spectrum radio quasars and OVV quasars could have higher $L_{\mathrm{BLR}}$ as required by Blazejowski et al. 38] in order to explain the $\gamma$-ray spectra for these objects. In our calculations we use $L_{\mathrm{UV}}=10^{46} \mathrm{erg} / \mathrm{s}$ and $\tau_{\mathrm{BLR}}=0.01$, which are typical values used for fitting the broad emission lines with photoionization models (for $\tau=0.01, L_{\mathrm{BLR}}=10^{44} \mathrm{erg} / \mathrm{s}$ ). For $\gamma$-ray attenuation by photon-photon pair production we need the photon number density per solid angle of BLR photons along the jet axis, $d n / d \Omega(\theta, z)=I(\theta, z) / c$ where $I(\theta, z)$ is the intensity. Calculation of the intensity is straightforward for distribution (i) where the BLR is a geometrically thin shell. In Fig. 3 we show the full angular dependence of the BLR $H_{\alpha}$ intensity at different distances $z$ along the jet for the case where the BLR is a thin shell at $r_{\mathrm{BLR}}=0.01 \mathrm{pc}$.

For distribution (ii) we shall calculate the intensity by integrating the emissivity along lines of sight through the BLR sketched in Fig. 4. A fraction $d \tau_{\mathrm{BLR}}=n_{\mathrm{cl}} \sigma_{\mathrm{cl}} d r$ of the central luminosity would then be re-processed in $r \rightarrow(r+d r)$. Since this spherical shell has volume $4 \pi r^{2} d r$ the emissivity (in $\operatorname{erg~s}^{-1} \mathrm{sr}^{-1} \mathrm{~cm}^{-3}$ ) of re-processed radiation inside the BLR at radius $r$ is

$$
j(r)=\frac{L d \tau_{\mathrm{BLR}}}{16 \pi^{2} r^{2} d r}=\frac{L n_{\mathrm{cl}} \sigma_{\mathrm{cl}}}{16 \pi^{2} r^{2}} .
$$

For $n_{\mathrm{cl}}=n_{0}\left(r / r_{i n, \mathrm{BLR}}\right)^{\alpha}$ and $R_{\mathrm{cl}}=R_{0}\left(r / r_{i n, \mathrm{BLR}}\right)^{\beta}$, where $n_{0}$ and $R_{0}$ is the number density and the radius of clouds at $r_{\text {in,BLR}}$, respectively, the optical depth of the entire BLR to central UV radiation is

$$
\tau_{\mathrm{BLR}}=\int_{r_{\mathrm{in}, \mathrm{BLR}}}^{r_{\mathrm{out}, \mathrm{BLR}}} n_{0} \pi R_{0}^{2}\left(\frac{r}{r_{i n, \mathrm{BLR}}}\right)^{\alpha+2 \beta} d r
$$

from which we obtain the constant $n_{0} R_{0}^{2}$ for any assumed value of $\tau_{\text {BLR }}$. Note that we shall use the power-law exponents preferred by Kaspi and Netzer [36], $\alpha=-1$ and $\beta=-1.5$. We treat separately the three possible emission region locations shown in Fig. 4 : (a) blob 1 inside the cavity inside the BLR, (b) blob 2 located within the BLR region, and (c) blob 3 


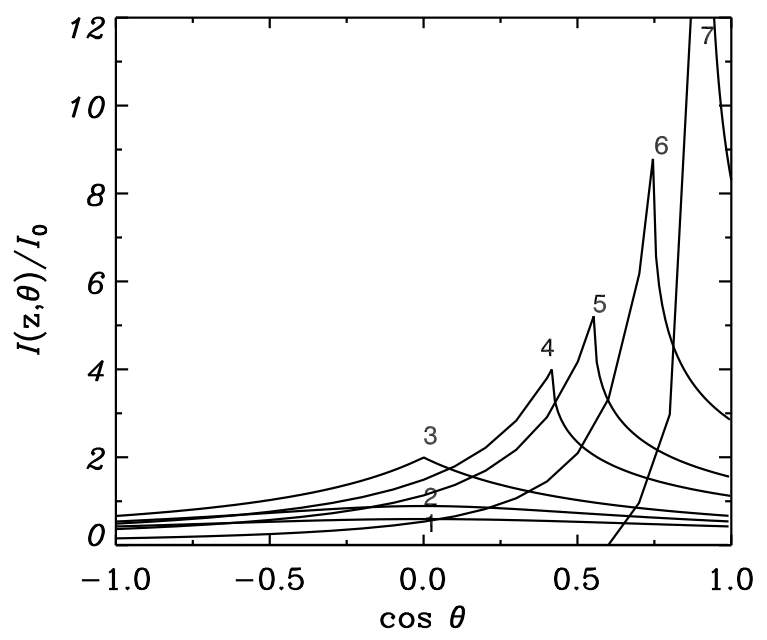

Figure 3: The angular distribution of the BLR $H_{\alpha}$ emission line intensity $I(z, \theta)$ for different distances from to the centre along the jet. The BLR is modeled as a geometrically thin shell at radius 0.01 pc. Labels $1-7$ correspond to $z=$ $0,0.008,0.009,0.01,0.011,0.012,0.015 \mathrm{pc}$, respectively. The anisotropy increases when the blob moves away from the BLR. The normalization factor $I_{0}$ is given by $I_{0}=c u_{0} / 4 \pi$, with $u_{0}=L_{\mathrm{BLR}} /\left(4 \pi z^{2} c\right)$. 


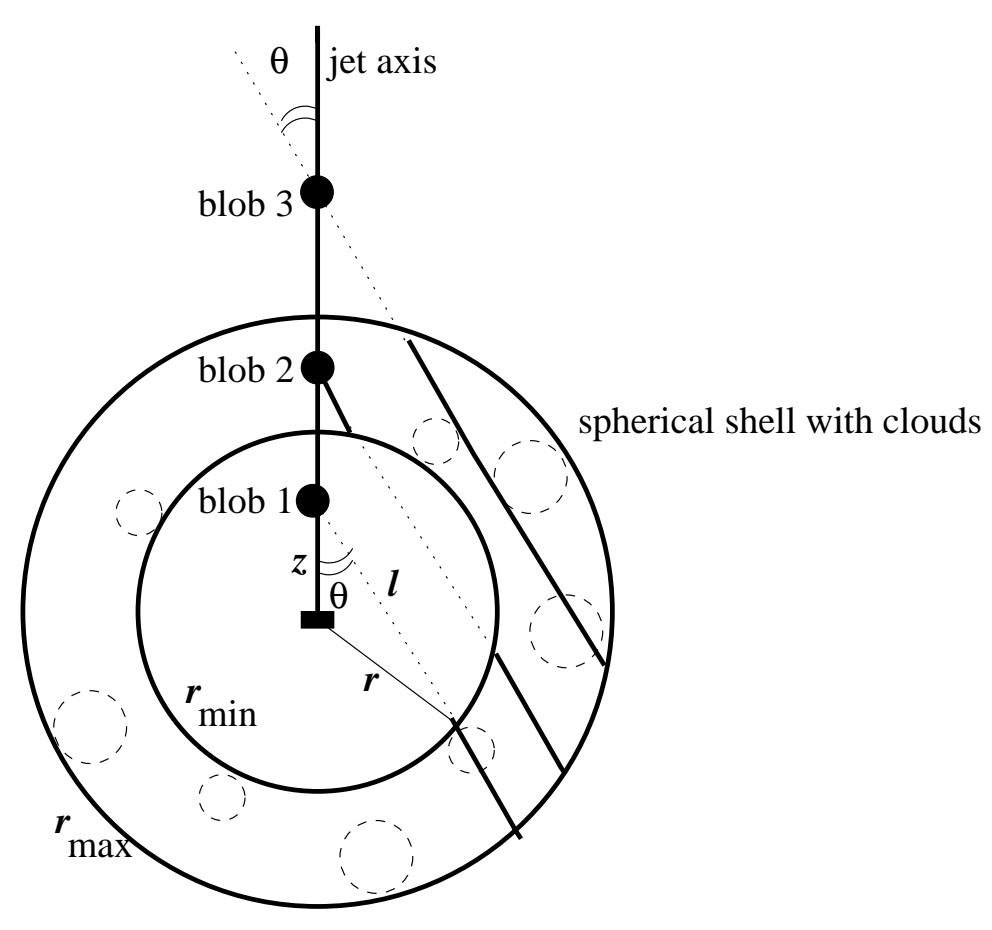

Figure 4: Sketch (not to scale) illustrating the geometry used for computation of the anisotropic intensity of the BLR radiation for distribution (ii) where the BLR is a geometrically thick spherical shell. The $\gamma$-ray emitting regions travel outwards along the jet axis and may be inside the cavity of the BLR (blob 1), within the BLR (blob2) or outside the BLR (blob 3), depending on their distance along the jet $z$.

is outside the BLR. The intensity of radiation at angle $\theta$ to the jet axis at a position $z$ within the BLR region (e.g. blob 2 in Fig. 4) is given by

$$
I(z, \theta)=\int_{0}^{l_{\min 1}} j(r) d l+\int_{l_{\min 2}}^{l_{\max }} j(r) d l,
$$

where

$$
\begin{gathered}
r^{2}=z^{2}-2 z l \cos \theta+l^{2} \\
l_{\max }=z \cos \theta+z\left(\cos ^{2} \theta+\left(\frac{r_{\text {out }, B L R}}{z}\right)^{2}-1\right)^{1 / 2}
\end{gathered}
$$

and

$$
l_{\min 1,2}=z \cos \theta \pm z\left(\cos ^{2} \theta+\left(\frac{r_{i n, B L R}}{z}\right)^{2}-1\right)^{1 / 2}
$$

The total (angle-integrated) energy density of BLR is:

$$
u(z)=\int \frac{I(z, \theta)}{c} d \Omega,
$$




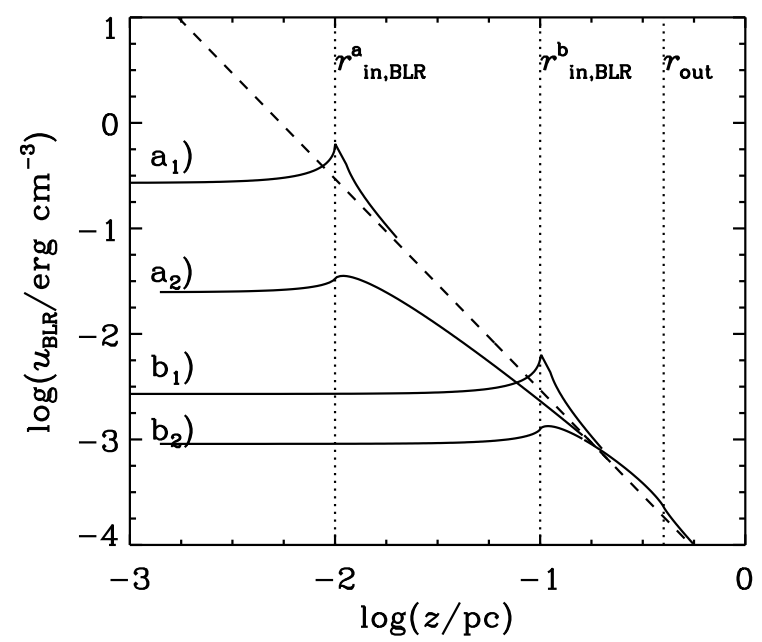

Figure 5: Energy density of radiation from BLR clouds at different distances $z$ along the jet axis. Cases labelled $\mathrm{a}_{1}$ and $\mathrm{a}_{2}$ are calculated for a BLR with $r_{\mathrm{BLR}}=0.01 \mathrm{pc}$ or $r_{\text {in,BLR }}=0.01$ pc. Cases labelled $\mathrm{b}_{1}$ and $\mathrm{b}_{2}$ correspond to a BLR with $r_{\mathrm{BLR}}=0.1 \mathrm{pc}$ or $r_{\mathrm{in}, \mathrm{BLR}}^{b}=0.1 \mathrm{pc}$. The label subscripts indicate the BLR cloud distribution used: $1-$ geometrically thin spherical shell (distribution i) $; 2$ - geometrically thick spherical shell (distribution ii) with $r_{\text {out,BLR }}=0.4 \mathrm{pc}, \alpha=-1$, and $\beta=-1.5$. The dashed line shows the crude approximation $u_{\mathrm{BLR}} \approx L_{\mathrm{BLR}} / 4 \pi c z^{2}$, with $L_{\mathrm{BLR}}=10^{44} \mathrm{erg} / \mathrm{s}$.

and is plotted in Fig. 5 as a function of distance along the jet. The energy density of BLR photons clearly depends also on the distribution of BLR clouds. At $z \gg r_{\mathrm{BLR}}$ (distribution i) and $z \gg r_{\text {out,BLR }}$ (distribution ii) the BLR photon energy density approaches the

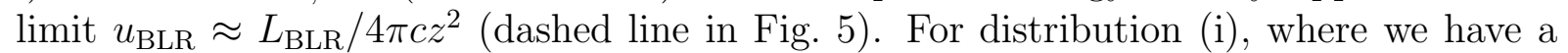
geometrically thin spherical shell for the BLR, the energy density peaks sharply at $r_{\text {BLR }}$ as found also by Ghisellini and Madau [5]. In the case of distribution (ii) the energy density peaks near $r_{\mathrm{in}, \mathrm{BLR}}$, but the curves are smoother as a result of the finite thickness of the BLR. Both cases are idealizations and there is probably a spread in cloud size at any given radius. From Fig. 5 we see that it is very important to know the right distribution of BLR clouds since there can be a difference of more than two orders of magnitude between the energy density of photons calculated for the same BLR luminosity but different BLR cloud distributions.

In Fig. 6 (a) we show the optical depth from various emission region heights, $z_{0}$, to infinity for absorption of $100 \mathrm{GeV}-100 \mathrm{TeV} \gamma$-rays in the radiation field of the BLR for distribution (i) where the BLR clouds are located in a geometrically thin shell at radius $0.01 \mathrm{pc}$. The optical depth is plotted for a BLR luminosity of $10^{44} \mathrm{erg} / \mathrm{s}$, but can be linearly scaled for other BLR luminosities. The anisotropy of the BLR radiation field is reflected in the shapes of the curves as one goes to higher $\gamma$-ray energies. Fig. 6(b) gives 

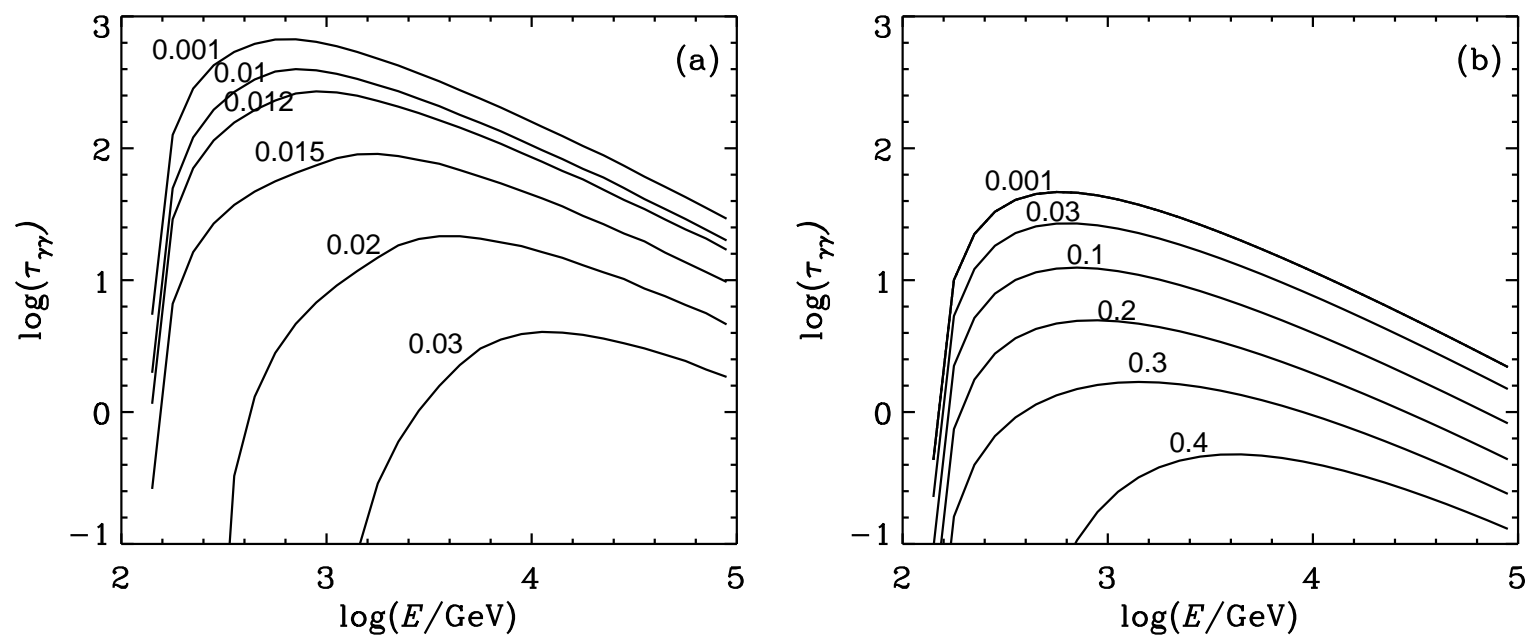

Figure 6: Photon-photon pair production optical depths for $\gamma$-rays traveling along the jet axis interacting with photons from a BLR having luminosity $L_{\mathrm{BLR}}=10^{44} \mathrm{erg} / \mathrm{s}$ and comprising: (a) a geometrically thin spherical shell of radius $r_{\mathrm{BLR}}=0.01 \mathrm{pc}$; (b) a geometrically thick spherical shell (distribution ii) with $r_{\mathrm{in}, \mathrm{BLR}}=0.01 \mathrm{pc}, r_{\mathrm{out}, \mathrm{BLR}}=$ $0.4 \mathrm{pc}, \alpha=-1$, and $\beta=-1.5$. Numbers attached to the curves give $z_{0}$ in pc.

the optical depth for cloud distribution (ii), with the inner radius at $0.01 \mathrm{pc}$ (the same as $r_{\mathrm{BLR}}$ in part a) and outer radius $0.4 \mathrm{pc}$. In this case, while at $z=0.01 \mathrm{pc}$ the optical depth is less than for distribution (i), $\tau_{\gamma \gamma}$ drops off more slowly with $z_{0}$, such that at only slightly larger distances the optical depth becomes orders of magnitude higher than for distribution (i) until $r_{\text {out,BLR }}^{b}$ is reached. This is in part because the possibility of near head-on collisions of $\gamma$-rays with BLR photons for $z<r_{\text {out,BLR }}^{b}$ for distribution (ii) - large angles between directions of interacting photons means more collisions will be above the pair production threshold.

Fig. 7 shows the $\gamma-\gamma$ opacity for $10 \mathrm{TeV}$ photons from $z=z_{0}$ to infinity vs. $L_{\mathrm{BLR}}$ for the two cases shown in Fig. 6. For blazars with a very low BLR luminosity, $10 \mathrm{TeV}$ photons can probably escape through the BLR. However, in quasars BLR typically have $L_{\mathrm{BLR}} \geq 10^{44} \mathrm{erg} / \mathrm{s}$, and so absorption of $10 \mathrm{TeV} \gamma$-rays can be quite important if the emission region is inside the BLR (distribution i) or is close to $r_{\text {in,BLR }}$ (distribution ii).

We comment here on a very intriguing problem, namely the absence of a Ly absorption edge in quasars 39. It was expected that BLR clouds situated along the line of sight should produce a sharp Ly absorption edge in more than $30 \%$ of quasars [40]. However, Maiolino et al. [39] have noted that none of the 101 quasars in the sample analyzed by Zheng et al. [41] show this feature. There are two possible explanations for the missing edge: (a) BLR clouds have a sufficiently large component of their random velocity along 


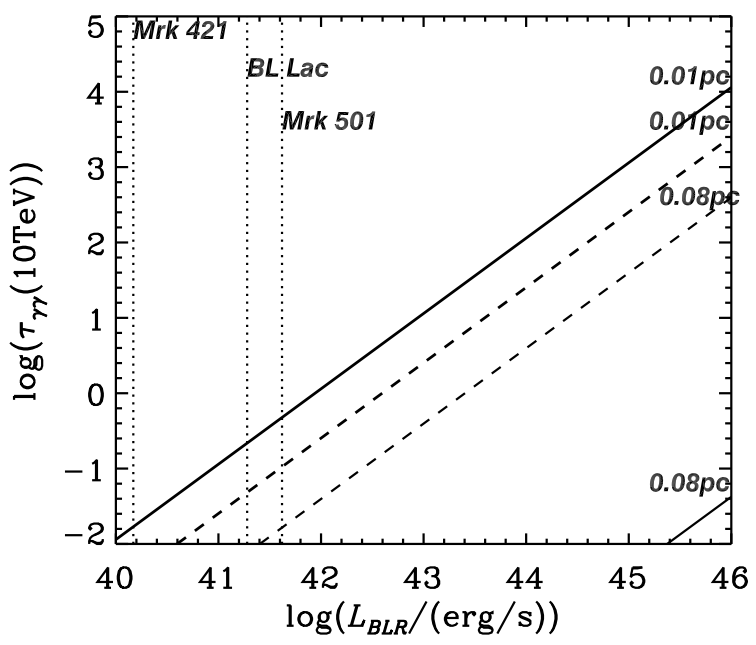

Figure 7: Photon-photon pair production optical depth for $10 \mathrm{TeV}$ photons from $z_{0}$ (numbers attached to the curves) to infinity for $L_{\mathrm{BLR}}=10^{44} \mathrm{erg} / \mathrm{s}$. Solid lines correspond to distribution (i) with $r_{\mathrm{BLR}}=0.01 \mathrm{pc}$, dashed to distribution (ii) with $r_{\mathrm{in}, \mathrm{BLR}}=0.01 \mathrm{pc}$, $r_{\text {out }, \mathrm{BLR}}=0.4 \mathrm{pc}, \alpha=-1$, and $\beta=-1.5$.

our line of sight to result in a broadening of the Ly edge and thereby making it difficult to observe, or (b) the BLR does not have a spherically symmetric distribution around the ionizing accretion disk, such that there are no BLR clouds intersecting our line of sight to the disk, and Maiolino et al. [39 suggest a flattened geometry could do this. Such a flattening in the cloud distribution could produce weaker densities of BLR photons and so would impede less the escape of $\mathrm{TeV}$ photons from the central region. However, we adopt a spherical distribution of clouds, and instead interpret the absence of an observed Ly absorption edge in 101 quasars to suggest that the BLR clouds must have a covering factor $f_{\text {cov }} \sim 0.03$ or less (95\% upper limit; for a covering factor 0.01 the probability of a BLR cloud being in the line of sight to the disk in none of 101 observations is $36 \%$, quite consistent with the observations). For a given covering factor, $f_{\text {cov }}$, the fraction of the disk luminosity intercepted by the BLR must then be less than $f_{\text {cov }}$, and justifies our use of $\tau_{\text {BLR }}=0.01$. Nevertheless, $\tau_{\text {BLR }} \sim 0.1$ have also been widely used, and would lead to photon-photon pair production opacities a factor of 10 higher than those presented here.

\section{Radiation from a dusty torus}

Chiaberge et al. 42 have suggested that tori do not exist in FR-I sources, or they must have a very thin geometry. This conclusion results from the correlation found between optical and radio cores of some FR-I sources. However, the sample used in this analysis 
represents only just under half of the FR-I galaxies tabled by Zirbel and Baum [43], and one needs additional observations of the polarized optical spectrum to reach a firm conclusion about this [44]. Nevertheless, these results could be interpreted as suggesting that perhaps there is a correlation between a flat infrared torus and a flat distribution of BLR clouds as suggested by Maiolino [39]. We do not know what mechanism, other than accretion, could cause such a flattening of the BLR and the torus. However, such a flattening might be understood in terms of a non-homogeneous spherical shell of BLR or dust in which the polar caps above and below the disk plane are much more diluted than the equatorial belt. Zier and Biermann [45] have shown that the caps of a spherical layer of dust could be diluted so much, that even a dense torus would have a doughnut shape.

We have already discussed some aspects related to the infrared radiation from ubiquitous dusty tori in quasars. Unification schemes for AGN generally involve the following components: Kerr black hole, a relativistic accretion disk, jets, broad line clouds and a dusty torus. For blazars the situation appears at first sight to be a little different, and we wonder why that might be. There are two issues which should be considered in connection with dusty tori in blazars. Firstly, since there is no direct evidence of any thermal emission from tori we should consider what indirect evidence (at any wavelength) there is about the existence of dust in the centres of host galaxies. Secondly, we should consider what kind of torus geometry would fit observations made at different wavelengths best.

Since unification schemes propose that FR-I and BL Lacs are related, FR-I sources being the mis-oriented counterparts of BL Lacs [14, we have searched the literature for information about dust structures associated with blazars/BL Lacs/FR-I. We note that Blazejowski et al. [38] required an external IR photon field from a torus scattered by relativistic jet electrons, in addition to those in the SSC model, in order to fit $\gamma$-ray spectra of OVV quasars observed by EGRET. A key to the existence of tori in blazars could come from establishing a link between the torus and the BLR. For example, when there is no detection of direct optical emission, the interpretation of spectro-polarimetric data on FR-I objects with strong evidence for infrared obscuration ( [15] and references therein) could suggest the existence of a BLR hidden by a thick torus. In this case, the observed polarized broad lines would be the result of scattering into the line of sight by free electrons in zones whose shape and orientation is determined by the torus' inner geometry. Hence, the detection of free electron scattering regions should be a good diagnostic of torus geometry. Falcke et al. [11] proposed that the opening angle of the torus might play a critical role in the FR-I and FR-II dichotomy - a closed torus covering a large fraction of $4 \pi$ steradians, as seen from the black hole, would obscure the internal activity of FR-I objects.

de Koff et al. [46] have discussed how the properties of the dust depend upon the radio properties of the object, and found that FR-II, which are powerful radio galaxies, have a rather clumpy dust distribution, and they suggest that this translates into a large opening angle of the torus. A less powerful radio jet (such as those in FR-I) would only slowly disperse the dust, and this type of obscuration might contribute to the deceleration of FR-I jets by entraining the material from the torus. 
We consider first the FR-I galaxy Centaurus A. Alexander et al. 33 explain the IR spectrum of Cen A using a combined model of infrared emission from startbursts, cirrus clouds and an AGN-type torus. A compact optically thick torus with $r_{\text {out,torus }}=3.6 \mathrm{pc}$ and an opening angle of $\phi=30^{\circ}$ would account for the observed flux. Centaurus A appears as a concentrated IR source in the $\mathrm{H}$ band [47] within a scale of tens of pc. No optical emission has been observed from the central source and, as Falcke et al. 11 suggested, this source may have a closed torus hiding the BLR which has not been detected even in polarized flux. Israel et al. [48], Rydbeck et al. [49] and Turner et al. [50] concluded that there is a dense nuclear torus with diameter $<230 \mathrm{pc}$. It is also interesting that Bryant et al. [47] show a clear picture of a warped dust lane obscuring the nuclear source - the radio jet being perpendicular to a large-scale torus which seems to be inclined with respect to the plane of the dust-lane.

Turning now to other FR-I radio galaxies, 3C218 (Hydra A) also shows evidence for a nuclear obscuration [51]. $3 \mathrm{C} 270$ is another FR-I radio galaxy for which a broad $H_{\alpha}$ emission line has been detected [52]. Ferrarese et al. [53] proposed that its nucleus is surrounded by a dust torus with a diameter of $120 \mathrm{pc}$ and optical depth $\tau \approx 1$. An inclination angle of $20^{\circ}$ between the axis of the torus and the line of sight would be in accordance with the detection of un-obscured nuclear optical emission [30].

Possible evidence against tori in blazars comes from the FR-I radio galaxy M87, which seems to have a very low IR flux which cannot be explained with a standard torus model [54]. The torus in this source could be very diluted, and heated less as a result of the extremely low accretion activity of M87 [13] such that its emission is dwarfed by the jet emission.

At kiloparsec scales, starburst activity dominates the infrared emission, but as one goes deeper into the nucleus of the galaxy, de Koff et al. [46] found that well organized dust structures were present in FR-I galaxies in the 3CR catalog, and this could indicate that the flow of matter towards the nucleus is rather steady in FR-I, allowing the formation of distinct torus features. These structures tended to be sharply defined small-scales disks with radii less than $\sim 2.5 \mathrm{kpc}$. The detection of large-scale dusty features (sometimes shaped as bars or dust-lanes) suggests that there could be an association between a smallscale infrared torus, such as those in Seyfert galaxies, and a large-scale torus sometimes identified with the dust structure of the galaxy. The structure of the nuclear torus could then depend on the large-scale dust distribution - well-organized kpc dust structures could extend inward towards smaller scales (small-scale torus surrounded by large-scale torus). We define this as a symbiosis between the large and small scale dusty features [16].

The above discussion leads us to the conclusion that dust could be present everywhere, even in FR-I objects where the torus cannot easily be detected. Since FR-I and BL Lacs are thought to be similar (apart from orientation) and there is some evidence of BLR and torus activity in FR-I, we postulate that the linkage between BLR and tori in quasars discussed by Chiaberge et al. [30] may apply also in blazars. We shall calculate next the optical depth for $\gamma-\gamma$ absorption in the infrared radiation field of tori.

We know that the IR emission from the torus is strongly related to the activity of the 


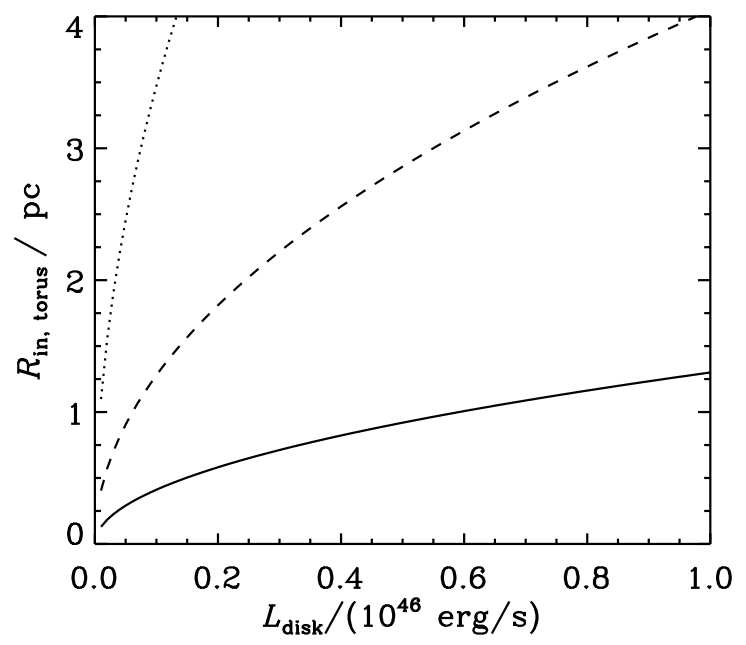

Figure 8: $R_{\text {in,torus }}$ vs. $L_{\text {disk }}$ for three different dust temperatures: $T=1500 \mathrm{~K}$ (solid curve), $1000 \mathrm{~K}$ (dashed curve), $700 \mathrm{~K}$ (dotted curve).

central object. The inner radius depends on $L_{\text {disk }}$, and is given by the sublimation radius of the dust [55]:

$$
R_{\text {in,torus }} \approx T_{1500}^{-2.8} L_{\text {disk, } 46}^{1 / 2} p c
$$

where $L_{\text {disk }}=10^{46} L_{\text {disk }, 46} \mathrm{erg} / \mathrm{s}$, and $T_{1500}$ is the dust temperature in units of $1500 \mathrm{~K}$ (the dust sublimation temperature is taken to be $1500 \mathrm{~K}$ ). The inner torus radius is plotted in Fig. 8 versus disk luminosity for three dust temperatures.

We start with a torus centred on the black hole, symmetric about the jet axis, and having a rectangular cross section with full height $h$, and inner and outer radii $R_{\text {in,torus }}$ and $R_{\text {out,torus. }}$. We shall discuss how $\tau_{\gamma \gamma}$ changes for different scales of tori, e.g., simulating an open torus by having either a rectangular cross section or a rectangular cross section with the inner edges cut away at angle $\phi$ (see Fig. 9).

As discussed earlier, in the context of the symbiosis between jets and accretion disks, an increase in jet power could be at the expense of disk luminosity. A lower disk luminosity at UV frequencies would reduce the heating of the inner surface of the dusty torus, causing the inner radius of the torus, given by Eq. 11, to be small (Fig. 8). This implies that fat dusty tori $\left(R_{\text {in,torus }} \ll h\right)$ could exist in AGN with very low central activity such as blazars, and this would be in accordance with the closed torus model discussed by Falcke et al. [11]. On the other hand, for a "cold torus" with $T<1000 \mathrm{~K}$, the inner radius would be far from the nucleus for a given luminosity (see Fig. 8). Such inefficient heating of the dust would probably describe best the torus in M87 which seems to be a peculiar object in that it has an extremely low mass accretion rate.

Due to its size, the energy density of the infrared photons remains fairly uniform inside the torus. A flat torus (i.e., $R \gg h$ ) could approximate the models proposed by Chiaberge 


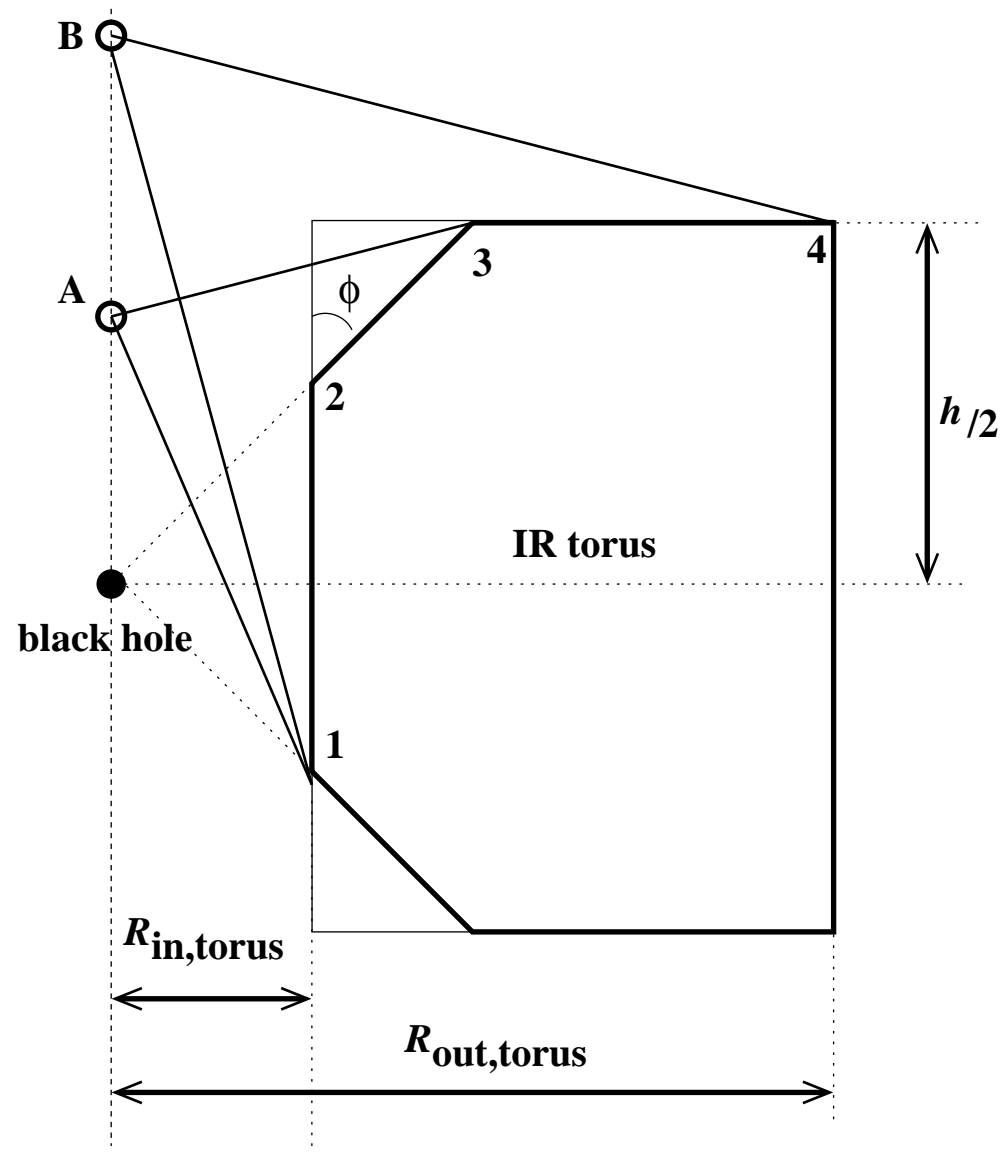

Figure 9: Torus cross-sections considered: rectangle, rectangle with edges cut away at angle $\phi$, the opening angle in this case (see text). $\gamma$-rays at point A interact with any photons emitted from the inner surface of the torus (between points 1 to 3). Gammarays at point $\mathrm{B}$ (above the torus) also interact with any photons emitted from the upper surface (between 3 to 4 ).

et al. [42]; see the discussion from the previous section and comments by Maiolino et al. [39] about a flattened BLR distributions. Although flat tori have not been detected, and we are uncertain about the stability of such a configuration of dust, we believe that it is an interesting possibility. For simplicity we model in this section a torus with a single black body temperature such that the IR intensity is constant within the solid angle subtended by the torus. We shall extend the work of Protheroe \& Biermann [17] for different torus geometries. Fig. 9 illustrates the geometry for interaction, at points A and B, of $\gamma$-rays with IR photons emitted from the surfaces of the torus.

Accretion rates are smaller in blazars than in quasars. Since the thickness of the torus is related to the accretion inflow and disk luminosity (through radiation pressure effects) [56] one expects tori in FR-I to be thinner, diluted, and/or less efficiently heated 


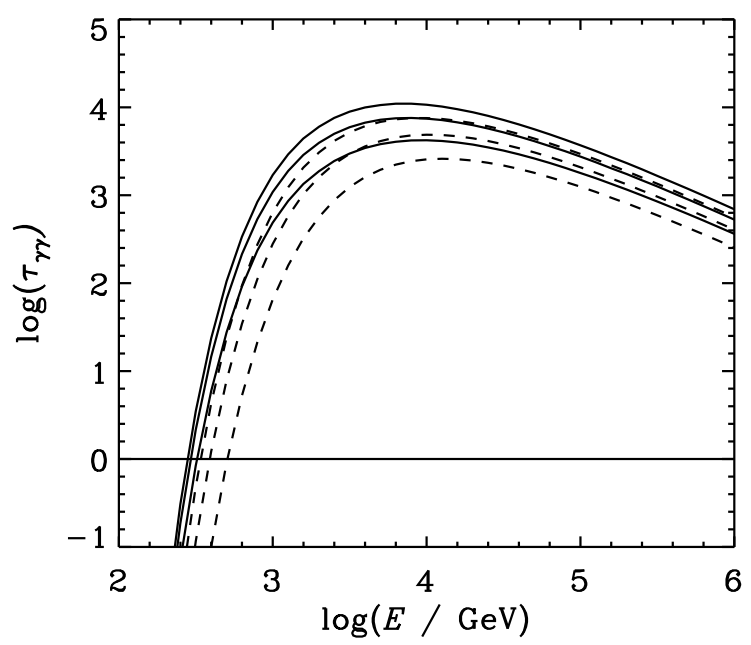

Figure 10: Optical depths for $\gamma$-rays traveling along the jet axis from $z=0$ to infinity in the torus IR radiation $(T=1000 \mathrm{~K})$ for $r_{\text {out }}=2 \mathrm{pc}$ and $r_{\text {in }}=0.1 \mathrm{pc}$ (solid curves) or $r_{\text {in }}=1$ pc (dashed curves). Results are show for three torus heights: $h=1$ pc (lower curves), $h=2$ pc (middle curves), $h=3$ pc (upper curves).

- as is probably the case in M87. Therefore, we have varied $h$ between 1 pc and 3 pc, $R_{\text {in,torus }}$ between $0.1 \mathrm{pc}$ and $1 \mathrm{pc}$, and $R_{\text {out,torus }}$ between $2 \mathrm{pc}$ and $10 \mathrm{pc}$. The optical depth from $z=0$ to infinity, for $\gamma$-rays traveling along the jet axis, is shown in Fig. 10 where we have compared the case of a torus with $R_{\text {in,torus }}=0.1 \mathrm{pc}$ (solid curves) with a torus having $R_{\text {in,torus }}=1 \mathrm{pc}$ (dashed curves) for three different torus heights $h$. We find that more $\gamma$-rays with energies around $10^{2.5-3.5} \mathrm{GeV}$ would be absorbed by $\gamma-\gamma$ interactions when the torus comes closer to the central source. We also find that an open torus geometry $(\phi>0)$ can significantly modify $\tau_{\gamma \gamma}$ as shown in Fig. 11(a) where it is again seen to have the greatest effect near the pair production threshold. If the torus has a large outer radius, then more IR photons from the upper surface of the torus are available for interaction with $\gamma$-rays along the jet. For example, for an extended torus with $R_{\text {out,torus }}=10 \mathrm{pc}$ the absorption of photons with energy above $2 \mathrm{TeV}$ is larger (compare dotted line with upper solid curve in Fig. 11a). As noted previously by Protheroe and Biermann [17], we can see that for photon energies above about several hundreds of $\mathrm{GeV}$ the opacity is large enough such that no $\mathrm{TeV} \gamma$-rays can emerge if the source is near the centre of the torus.

Risaliti et al. [57 have shown that a torus is stable if the mass of the dust in the torus does not exceed the dynamical mass flowing toward the center. This constrains the outer torus radius, $R_{\text {out,torus }}$, to have values less than $10 \mathrm{pc}$ for a column density of dust along the line of sight that intersects the obscuring medium of $N \approx 10^{24} \mathrm{~cm}^{-2}$. FR-I objects could have diluted tori with column densities of $N \approx 10^{22} \mathrm{~cm}^{-2}$, or lower, but still be optically thick, and this would impose a upper limit of $\sim 100 \mathrm{pc}$ for the torus' 

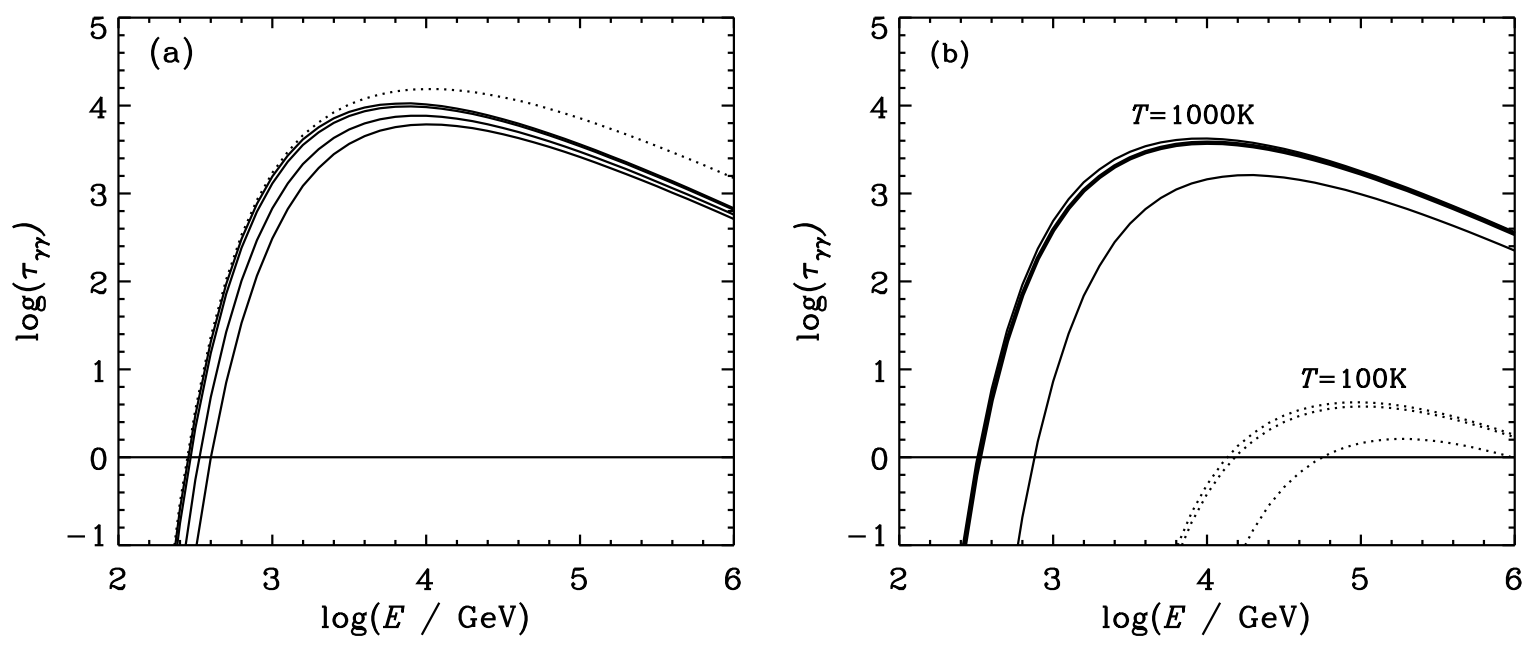

Figure 11: Optical depths for $\gamma$-rays traveling along the jet axis from $z=z_{0}$ to infinity in the torus IR radiation. (a) $T=1000 \mathrm{~K}, z_{0}=0, r_{\text {in }}=0.1 \mathrm{pc}, r_{\text {out }}=2 \mathrm{pc}, h=3 \mathrm{pc}$ and with the torus inner edge cut away at angle $\phi=0^{\circ}$ (upper solid curve), $10^{\circ}, 30^{\circ}$, $50^{\circ}$ (lower solid curve). Dotted curve is for $r_{\text {out }}=10 \mathrm{pc}$ and $\phi=0^{\circ}$. (b) $r_{\text {in }}=0.1 \mathrm{pc}$, $r_{\text {out }}=2 \mathrm{pc}, h=1 \mathrm{pc}, \phi=0^{\circ}, T=1000 \mathrm{~K}$ (solid curves) or $T=100 \mathrm{~K}$ (dotted curves), for $z_{0}=0.01 \mathrm{pc}$ (upper curves), $0.1 \mathrm{pc}$ (middle curves), and $0.5 \mathrm{pc}$ (lower curves) 
outer radius. However, Maiolino et al. [58 interpret their data as suggesting that the inner parts of tori are much denser than the outer parts, and find a gradient in the torus' covering factor that would give $R_{\text {out,torus }}<20$ pc for Seyfert galaxies. The covering factor plays a crucial role in these models, as well as the gas mass enclosed within the torus [57]. For simplicity, we take a value of $10 \mathrm{pc}$ as the maximum radius of the dust torus which could be relevant to our analysis.

In Fig. 11(b) we show how the optical depth depends on the distance of $\gamma$-ray emission region along the jet. So far, we have assumed that the torus radiates as a perfect black body. However, if the torus is patchy or not optically thick at IR wavelengths we may have diluted black body radiation, $u_{\mathrm{IR}} \approx \eta_{\mathrm{IR}} a T^{4} \mathrm{erg} / \mathrm{cm}^{3}$, where $a$ is the radiation constant. All our curves would be multiplied by $1 / \eta_{\mathrm{IR}}$ in this case. Alternatively, for a poor heating mechanism related to a low luminosity of the accretion disk the dust could be heated to lower temperatures, e.g. $T \approx 100 \mathrm{~K}$, and this results in reducing the $\mathrm{GeV}$ to $\mathrm{TeV}$ opacity considerably, with the pair production threshold $\gamma$-ray energy increasing as $\sim 1 / T$ as illustrated in Fig. 11(b) by the dotted curves. We note however, that Blazejowski et al. [38] require $\eta_{I R} \approx 0.3$ to fit $\gamma$-ray spectra of OVV quasars with an external inverse Compton model, and this still allows for significant $\mathrm{TeV} \gamma$-ray absorption in this type of blazar. Extremely low values of $\eta_{I R}$ may apply in objects such as M87.

\section{Constraints on the position of the $\gamma$-ray emitting region and conclusions}

The ADJ model gives a simplified approach to the symbiosis between disks and jets. It could explain the low central activity in some blazars, and for very low mass accretion rates an ADJ could transform into an EDAF or an ADAF. The strong variability observed in blazars from radio to $\gamma$-ray frequencies could be a consequence of the feeding mechanism of the jet at the boundary layer. A local perturbation produced in the boundary layer, such as a rapid variation of $R_{\text {jet }}$ or $\dot{M}$, would propagate through the nozzle and along the jet causing transient phenomena. As the jet extracts more energy from the disk, this could leave less energy to be dissipated in the disk as radiation. The jet/disk symbiosis then mainly modifies the energetics in the central parsec of AGN: increasing the power of the jet could weaken the disk which therefore would not efficiently ionize the BLR clouds or efficiently heat the dust in the torus, and so would affect important target photon fields for interactions of $\gamma$-rays (and also accelerated electrons and protons) along the jet.

Fig. 12 summarizes our results in a way that makes it easy to see the relative importance of the three radiation fields to the absorption of high energy $\gamma$-rays. We show the optical depth as a function of height of the emission region above the plane of the disk for three $\gamma$-ray energies spanning the range currently explored with optical Cherenkov telescopes: (a) $0.5 \mathrm{TeV}$ which is close to the threshold of present telescopes, (b) $2.5 \mathrm{TeV}$, (c) 12.5 TeV typically observed during flaring activity in nearby blazars. The shaded areas give the range in optical depth for the models discussed earlier, but normalized for an ac- 
cretion disk luminosity $L_{\text {disk }}=10^{46} \mathrm{erg} \mathrm{s}^{-1}$, a broad line region luminosity $L_{\mathrm{BLR}}=10^{44} \mathrm{erg}$ $\mathrm{s}^{-1}$, and torus IR dilution factor $\eta_{\mathrm{IR}}=1$. We see that at all three energies, all three radiation fields can play a very important role. We shall consider each radiation field in turn, first for quasars and then for blazars.

In quasars, with typically $L_{\text {disk }} \geq 10^{46} \mathrm{erg} \mathrm{s}^{-1}$ the accretion disk radiation will surely cut off $\gamma$-rays above $0.5 \mathrm{TeV}$ if the emission region is below $\sim 0.005-0.03 \mathrm{pc}$ (depending on $\dot{m}$ ). At $2.5 \mathrm{TeV}$ and $12.5 \mathrm{TeV}$ the corresponding minimum emission region heights to avoid absorption in quasars are $\sim 0.02-0.08 \mathrm{pc}$ and $\sim 0.03-0.2 \mathrm{pc}$. The BLR is active in quasars, and is likely to have $L_{\mathrm{BLR}} \geq 10^{44} \mathrm{erg} \mathrm{s}^{-1}$ such that the emission region would have to be above $0.03-0.3 \mathrm{pc}$ (depending on BLR cloud distribution) at all three energies. For a luminous disk as in quasars, one would expect an active torus to cut off TeV energy $\gamma$-rays unless the emission region were well above the torus: $z_{0}>0-2 \mathrm{pc}(0.5 \mathrm{TeV}$, note $\tau_{\gamma \gamma}^{\mathrm{IR}}$ can be small at the energy), $z_{0}>2-10 \mathrm{pc}(2.5 \mathrm{TeV})$, and $z_{0}>4-30 \mathrm{pc}(12.5 \mathrm{TeV})$, depending on torus geometry. All known quasars are at a distance such that multi-TeV $\gamma$-rays are expected to be absorbed in the infrared background radiation during their propagation to Earth. However, observations at energies below $1 \mathrm{TeV}$ are possible with current atmospheric Cherenkov telescopes, and multi-GeV observations will be made with GLAST [59] within a few years. Future measurements of the shape of the $\gamma$-ray spectrum of quasars at the cut-off might have something to say about which radiation field is responsible for the cut-off, and hence the location of the emission region, although this is likely to be very difficult (compare the optical depth vs. energy in the disk radiation, BLR radiation and torus radiation in Figs. 2, 6 and 11).

The situation is somewhat different in blazars. Here, we can have significantly less powerful accretion disks than in quasars. For example, to avoid attenuation of $\gamma$-rays in the disk radiation for a disk luminosity of $10^{44}-10^{46} \mathrm{erg} \mathrm{s}^{-1}$ one would need to be above $\sim 0.001-0.005 \mathrm{pc}(0.5 \mathrm{TeV})$, and above $\sim 0.005-0.03 \mathrm{pc}(>2.5 \mathrm{TeV})$, depending on $\dot{m}$. Note that at all energies the optical depth in the BLR field becomes less than unity if the BLR luminosity is lower than $10^{42} \mathrm{erg} \mathrm{s}^{-1}$. However, for $L_{\mathrm{BLR}}=10^{42}-10^{44} \mathrm{erg} \mathrm{s}^{-1}$ the $\gamma$-ray emission region would need to be above $0.03-0.3 \mathrm{pc}$ (depending on BLR cloud distribution), as for quasars, to avoid attenuation. In the case of the torus radiation, if blazars have active tori, they could present a serious problem for the escape of $\mathrm{TeV} \gamma-$ rays from emission regions which are not well above the torus (as in the case of quasars). However, because of the low disk luminosity, the torus' inner radius would probably be small ( $\sim 0.1 \mathrm{pc}$ for $L_{\text {disk }} \geq 10^{44} \mathrm{erg} \mathrm{s}^{-1}$, see Eq. 11), and the torus would be likely to be small, generally, and the optical depth would probably be at or below the lower boundary of that shown. Inefficient heating could also result in dilution of the torus radiation $\left(\eta_{\mathrm{IR}}<1\right)$ or in a lower temperature, and consequently lower optical depths (see e.g. Fig. 11b).

We have demonstrated that the external radiation fields present within the parsec scale of AGN may create problems for the escape of high energy $\gamma$-rays. The effect is dramatic for quasars where the densities of external radiation fields are much higher than in blazars. The symbiosis between the accretion disk and jets could result in lower radiation fields 

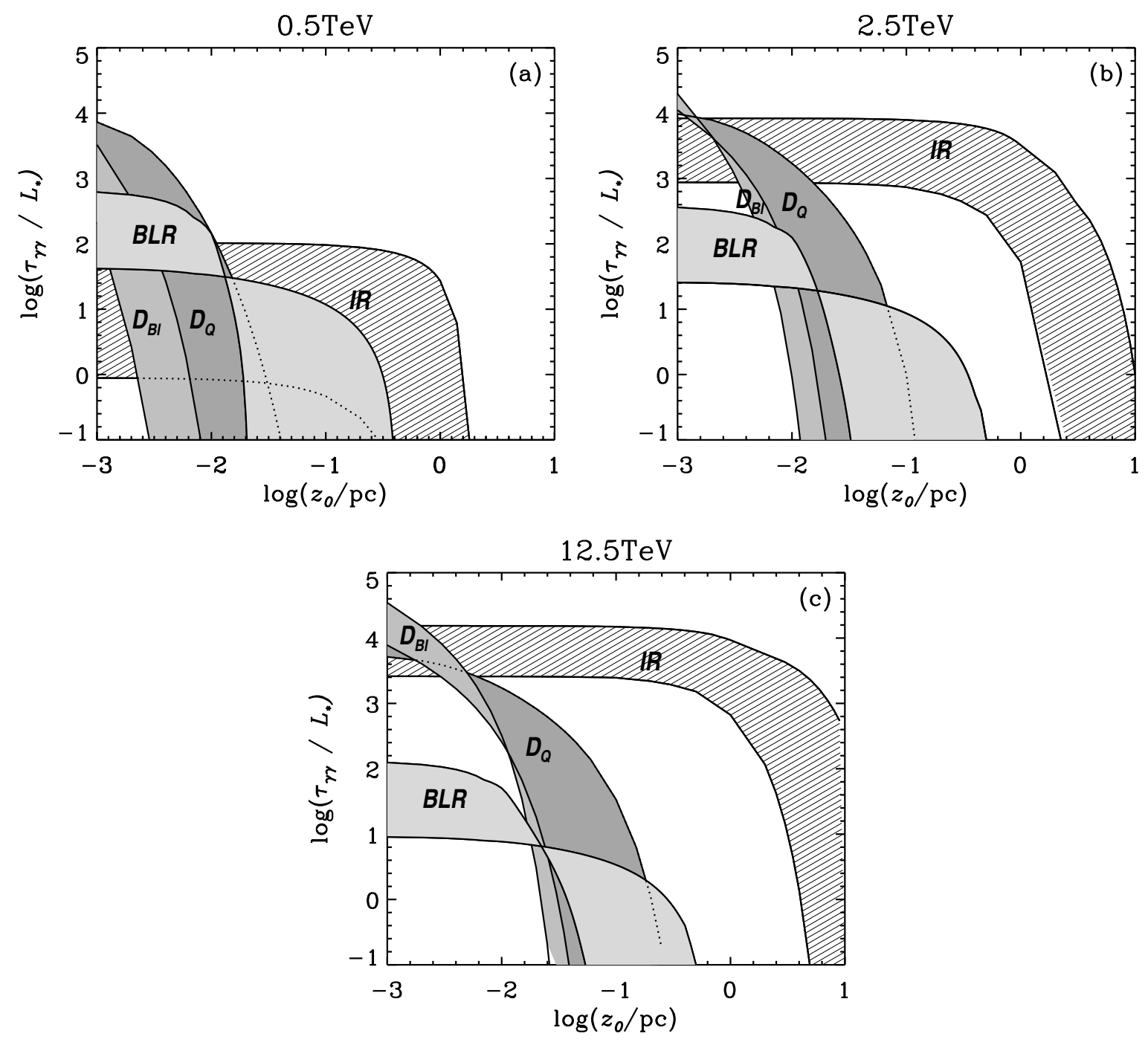

Figure 12: Photon-photon optical depth, divided by $L *$, vs. height of the emission region $z_{0}$ above the disk for interaction with BLR photons ("BLR", $L *=L_{\mathrm{BLR}} / 10^{44} \mathrm{erg}$ $\mathrm{s}^{-1}$ ), IR photons ("IR", $L * \equiv \eta_{\mathrm{IR}}$ ) and accretion disk photons in blazars ("D $\mathrm{Bl}$ ", $L *=L_{\text {disk }} / 10^{46} \mathrm{erg} \mathrm{s}^{-1}$ ) and quasars ("D ${ }_{\mathrm{Q}}$ ", $L *=L_{\text {disk }} / 10^{46}$ erg $\mathrm{s}^{-1}$ ). The ranges of model parameters used for the three radiation fields are: accretion disk radiation - as in Fig. 2b; BLR $-\tau_{\mathrm{BLR}}=0.01, R_{\mathrm{BLR}}=0.01 \mathrm{pc}$ (upper boundary on left is as in Fig. 6a), $R_{\text {in,BLR }}=0.01 \mathrm{pc}, R_{\text {out }, \mathrm{BLR}}=0.4 \mathrm{pc}$ (lower boundary on left is as in Fig. $6 \mathrm{~b}$ ); torus $T=1000 \mathrm{~K}, \phi=0$, upper curve $\left(h=3 \mathrm{pc}, r_{\text {in }}=0.1 \mathrm{pc}, r_{\text {out }}=10 \mathrm{pc}\right)$, lower curve $\left(h=1 \mathrm{pc}, r_{\mathrm{in}}=1 \mathrm{pc}, r_{\text {out }}=2 \mathrm{pc}\right)$. 
around the base of the jet during flaring activity if the jet takes a higher fraction of the matter entering the disk, and this could minimize the dramatic effect of the $\gamma$-ray absorption. However, although heated by the disk, the torus IR field would not react rapidly to changes in disk luminosity because of its larger scale, and so the escape of $\mathrm{TeV}$ $\gamma$-rays would require the emission region to be located at parsec distances from the black hole, far above the torus unless the torus is very inefficiently heated although even in this case one can expect some attenuation. This is in accordance with conclusion of Protheroe and Biermann [17], and also the recent work of Jorstad et al. [60] who concluded that the temporal connection between the radio and $\gamma$-ray variations suggests that the $\gamma$-rays appear to originate far away from the radio core.

In conclusion, the origin of the $100 \mathrm{GeV}-1000 \mathrm{GeV} \gamma$-ray photons must be above the inner part of BLR region for any quasar. Blazars usually have low central luminosities, and this translates into a having a low BLR luminosity, and possibly a closed torus. Mrk 421 seems to have a BLR with an extremely low luminosity $L_{\mathrm{BLR}} \approx 1.5 \times 10^{40} \mathrm{erg} / \mathrm{s}$ [27] and so could be transparent to $\mathrm{TeV} \gamma$-rays. However, $\mathrm{TeV} \gamma$-rays would need to be produced far above the torus unless the torus is very inefficiently heated. Farther along the jet external radiation fields become less important. In this case the radiation produced inside the emission region in the jet (e.g. synchrotron emission) becomes the dominant radiation field for photon-photon pair production by $\gamma$-rays produced in the jet.

\section{Acknowledgments}

We thank Peter Biermann and Geoff Bicknell for useful discussions. This work was supported by an Australian Research Council grant to RJP.

\section{References}

[1] C. Dermer, S. Sturner, R. Schlickeiser, ApJ 416 (1993) 458.

[2] M. Sikora, M.C. Begelman, M.J. Rees, ApJ 421 (1994) 153.

[3] K. Mannheim, A\&A 297 (1995) 321.

[4] R.J. Protheroe, IAU Colloquium 163 ASP Conf. Series 121 ed. D.T. Wickramasinghe et al. (1997) 585.

[5] G. Ghisellini, P. Madau, MNRAS 280 (1996) 67.

[6] W. Bednarek, R.J. Protheroe, MNRAS 310 (1999) 577.

[7] F.W. Stecker, O.C. De Jager, A\&A 334 (1998) L85.

[8] R.J. Protheroe, H. Meyer, Phys. Letters B 493 (2000) 1. 
[9] A.-C. Donea, P.L. Biermann, A\&A 316 (1996) 43.

[10] M.A. Malkan, W.L. Sargent, ApJ 254 (1982) 22.

[11] H. Falcke, Gopal-Krishna, P.L. Biermann, 1995 A\&A 298 (1995) 395.

[12] I.D. Novikov, K.S. Thorne, Black Hole Astrophysics, DeWitt C., DeWitt B., Gordon \& Breach, New York, 1973, 343.

[13] C.S. Reynolds, T. Di Matteo, A.C. Fabian, U. Hwang, C.R. Canizares, MNRAS 283 (1996) L111.

[14] C.M. Urry, P. Padovani, PASP 107 (1995) 803.

[15] Antonucci, R., (2001) astro-ph/0103048.

[16] A.-C. Donea, R.J. Protheroe, PASA 19 (2002) to appear.

[17] R.J. Protheroe, P.L. Biermann Astropart. Phys. 6 (1997) 293.

[18] R.D. Blandford, R.L. Znajek, MNRAS 179 (1997) 433.

[19] H. Falcke, M.A. Malkan, P.L. Biermann, A\&A 298 (1995) 375.

[20] A.-C. Donea, P.L. Biermann, PASA 19 (2002) to appear.

[21] A.-C. Donea, R.J. Protheroe, Astropart. Phys., (2002), to be submitted

[22] A.-C. Donea, J.-L. Masnou, F. Donea, Proceeding conf. High Energy Gamma-Ray Astronomy, Heidelberg 2000, ed. Aharonian and Völk, American Institute of Physics, Melville, New York 558 (2001) 708

[23] A.-C. Donea, H. Falcke, P.L Biermann, "The Central Parsecs of the Galaxy", eds. H.Falcke, A.Cotera, W.Duschl, F.Melia, M.Rieke, ASP Conf. Series 186 (1999) 162.

[24] R. Narayan, I. Yi, ApJL 428 (1994) L13.

[25] B.M. Peterson, P. Berlind, R. Bertram, et al., ApJ 425(1994) 622.

[26] E.A. Corbett, A. Robinson, D.J. Axon, J.H. Hough, MNRAS 311 (2000) 485.

[27] R. Morganti, M.-H. Ulrich, C. N. Tadhunter, MNRAS 254 (1992) 546.

[28] A. Celotti, A.C. Fabian, M.J. Rees, MNRAS 293 (1998) 239.

[29] M. Stitckel, J. Fried, H. Kühr, A\&AS 98 (1993) 393.

[30] M. Chiaberge, A. Celotti, A. Capetti, G. Ghisellini, A\&A 358 (2000) 104. 
[31] J.H. Krolik, C.F. McKee, C.B. Tarter, ApJ 249 (1981) 422.

[32] J. Baldwin, G. Ferland, K. Korista, D. Verner, ApJL 455 (1995) L119.

[33] D.M. Alexander, A. Efstathiou, J.H. Hough, D.K. Aitken, D. Lutz, P.F. Roche, E.A. Sturm, MNRAS 310 (1999) 78.

[34] S.D. Murray, D.T. Woods, J.I. Castor, R.I. Klein, C.F. McKee, ApJ 454 (1995) 133L.

[35] M.J. Fromerth, F. Melia, ApJ 549 (2001) 205.

[36] S. Kaspi, H. Netzer, ApJ 524 (1999) 71.

[37] A. Celotti, P. Padovani, G. Ghisellini, MNRAS 286 (1997) 415.

[38] M. Blazejowski, M. Sikora, R. Moderski, G.M. Madejski, ApJ 545 (2000) 107.

[39] R. Maiolino, M. Salvati, A. Marconi, R.R.J. Antonucci, A\&A 375 (2001) 25.

[40] R.R.J. Antonucci, A.L. Kinney, H.C. Ford, ApJ 342 (1989) 64.

[41] W. Zheng, G.A. Kriss, R.C. Telfer, J.P. Grimes, A.F. Davidsen, ApJ 475 (1997) 469.

[42] M. Chiaberge, A. Capetti, A. Celloti, A\&A 349 (1999) 77.

[43] E.L. Zirbel, S.A. Baum, ApJ 448 (1995) 521.

[44] D. Whysong, R.R.J. Antonucci, submitted ApJL (2001) astro-ph/0106381.

[45] C. Zier, P.L. Biermann, A\&A, 377 (2001) 23.

[46] S. de Koff, P. Best, S.A. Baum, et al., ApJS 129 (2000) 33.

[47] J.J. Bryant, R.W. Hunstead, MNRAS 308 (1999) 431.

[48] F.P. Israel, E.F. van Dishoeck, et al., A\&A 227 (1990) 342.

[49] G. Rydbeck, T. Wiklind, M. Cameron, W. Wild, A. Eckart, R. Genzel, H. Rothermel, A\&A 270 (1993) 3.

[50] T.J. Turner, I.M. George, K. Nandra, R.F. Mushotzky, ApJ. 488 (1997) 164.

[51] R.M. Sambruna, G. Chartas, M. Eracleous, R.F. Mushotzky, ApJ 532 (2000) 91.

[52] A.J. Barth, A.V. Filippenko, ApJ 525 (1999) 673.

[53] L. Ferrarese, H.C. Ford, ApJ 515 (1999) 583.

[54] E. S. Perlman, W.B. Sparks, et al., ApJ 561 (2001) L51. 
[55] Barvainis, R. ApJ. 320 (1987) 537.

[56] M.A. Dopita, C. Heisler, S. Lumsden, J. Bailey, ApJ 498 (1998) 570.

[57] G. Risaliti, R. Maiolino, M. Salvati, ApJ 522 (1999) 157.

[58] R. Maiolino, A. Krabbe, N. Thatte, R. Genzel, ApJ 493 (1998) 650.

[59] N. Gehrels, P. Michelson, Astropart. Phys. 11 (1999) 277.

[60] S.G. Jorstad, A.P. Marscher, J.R. Mattox, M.F. Aller, H.D. Aller, A.E. Wehrle, S.D. Bloom, ApJ 556 (2001) 738. 